\title{
Novel Prognostic Nomograms for Hepatocellular Carcinoma Patients with Microvascular Invasion: Experience from a Single Center
}

\author{
Liangliang X ${ }^{1}$, Lian $\mathrm{Li}^{1}$, Peng Wang ${ }^{1}$, Ming Zhang ${ }^{1}$, Yanfang Zhang ${ }^{2}$, Xiangyong Hao ${ }^{3}$, Lvnan Yan ${ }^{1}$, Bo Li ${ }^{1}$, Tianfu Wen ${ }^{1}$, and \\ Mingqing $\mathrm{Xu}^{1}$ \\ ${ }^{1}$ Department of Liver Surgery, ${ }^{2}$ Center of Infectious Diseases, West China Hospital, Sichuan University, Chengdu, and ${ }^{3}$ Department of General \\ Surgery, Gansu Provincial Hospital, Lanzhou, China
}

Background/Aims: Microvascular invasion (MVI) is an established risk factor for hepatocellular carcinoma (HCC). However, prediction models that specifically focus on the individual prognoses of HCC patients with MVI is lacking. Methods: A total of $385 \mathrm{HCC}$ patients with MVI were randomly assigned to training and validation cohorts in a 2:1 ratio. The outcomes were disease-free survival (DFS) and overall survival (OS). Prognostic nomograms were established based on the results of multivariate analyses. The concordance index (C-index), calibration plots and Kaplan-Meier curves were employed to evaluate the accuracy, calibration and discriminatory ability of the models. Results: The independent risk factors for both DFS and OS included age, tumor size, tumor number, the presence of gross vascular invasion, and the presence of Glisson's capsule invasion. The platelet-tolymphocyte ratio was another risk factor for OS. On the basis of these predictors, two nomograms for DFS and OS were constructed. The $\mathrm{C}$-index values of the nomograms for DFS and OS were 0.712 (95\% confidence interval [Cl], 0.679 to $0.745 ; p<0.001)$ and $0.698(95 \% \mathrm{Cl}, 0.657$ to 0.739 ; $\mathrm{p}<0.001)$, respectively, in the training cohort and 0.704 (95\% $\mathrm{Cl}, 0.650$ to $0.708 ; \mathrm{p}<0.001)$ and $0.673(95 \% \mathrm{Cl}, 0.607$ to 0.739 ; $p<0.001)$, respectively, in the validation cohort. The calibration curves showed optimal agreement between the predicted and observed survival rates. The Kaplan-Meier curves suggested that these two nomograms had satisfactory discriminatory abilities. Conclusions: These novel predictive models have satisfactory accuracy and discriminatory abilities in predicting the prognosis of HCC patients with MVI after hepatectomy. (Gut Liver 2019;13:669-682)

Key Words: Carcinoma, hepatocellular; Microvascular inva- sion; Hepatectomy; Prognosis; Nomogram

\section{INTRODUCTION}

As the most common primary (70\% to 90\%) liver cancer, hepatocellular carcinoma (HCC) is the fifth most frequent malignancy and the second leading cause of cancer-related mortality all over the world. ${ }^{1}$ Annually, approximately seven million new cases of HCC were diagnosed and almost an equal number of deaths occurred. ${ }^{1}$ With the high prevalence of hepatitis B virus (HBV) infection, more than 50\% of the total number of new cases and deaths occurred in China. ${ }^{1}$ Since the limited availability of donor organs and expensive medical cost make liver transplantation problematic, liver resection is still considered to be the first-line option for the patients with early stage HCC. ${ }^{2-4}$ Moreover, increasing evidence has confirmed that the patients with intermediate stage HCC or even advanced HCC with portal vein tumor thrombosis limited to the first-order branch could also achieve survival benefit from liver resection. ${ }^{5,6}$ However, the long-term survival of HCC remains unsatisfactory owing to the extremely high postoperative recurrence rate.,8

Microvascular invasion (MVI) is defined as the presence of tumor emboli in the hepatic veins, portal system, and/or lymphatic ducts on microscopy. ${ }^{9}$ The incidence of MVI ranged from $15.0 \%$ to $57.1 \%$ in previous studies. ${ }^{9}$ The detection rate of MVI was closely correlated with the tumor size and numbers. ${ }^{10}$ The presence of MVI has been widely recognized as a poor prognostic factor for patients who undergo curative liver resection., ${ }^{911-14}$ And for the patients with MVI, deeper vascular-wall invasion, more invaded vessels and further distance of invasion indicated worse prognostic outcomes. ${ }^{10,15}$

In recent years, many studies have identified multiple clini-

\footnotetext{
Correspondence to: Mingqing Xu

Department of Liver Surgery, West China Hospital, Sichuan University, No. 37 Guo Xue Xiang, Chengdu 610041, China

Tel: +86-18980601373, Fax: +86-02885422055, E-mail: xumingqing0018@163.com

Received on November 4, 2018. Revised on December 26, 2018. Accepted on December 28, 2018. Published online July 19 , 2019.

pISSN 1976-2283 eISSN 2005-1212 https://doi.org/10.5009/gnl18489

Liangliang $\mathrm{Xu}$, Lian Li, and Peng Wang contributed equally to this work as first authors.

(a) This is an Open Access article distributed under the terms of the Creative Commons Attribution Non-Commercial License (http://creativecommons.org/licenses/by-nc/4.0)

which permits unrestricted non-commercial use, distribution, and reproduction in any medium, provided the original work is properly cited.
} 
cal variables associated with MVI, and some investigators even established accurate models for predicting MVI based on preoperative parameters. ${ }^{11,12,16}$ These studies were meaningful to guide the surgeons to choose reasonable treatments and expand the resection margin, owing to the fact that more than 90\% MVI occurred in the area where was less than $1 \mathrm{~cm}$ from the tumor edge. ${ }^{10}$ However, to the best of our knowledge, studies have seldomly specifically focused on the prognosis of the patients who were histologically diagnosed with MVI after surgery so far. And the independent risk factors which were associated with the recurrence and long-term survival of this subset of patients remain unclear. Furthermore, as MVI itself is an established risk factor, it is uncertain whether conventional staging systems of HCC were appropriate for this special group patients or not. Therefore, an effective prediction model is imperative to plan personalized surveillance strategies and adjuvant therapies after surgery for this subset of patients. Nomogram has been regarded as a reliable tool to integrate and quantify important risk factors for oncologic prognosis. ${ }^{17-19}$ And its superiority could be estimated by comparing the concordance index (C-index) with other staging systems. Using this easy-to-use predicting model, it is convenient to calculate the unique risk scores and an individual survival possibility for each patient. The aim of this study was to generate novel nomograms for the prognostic prediction of HCC patients with MVI after curative hepatectomy.

\section{MATERIALS AND METHODS}

\section{Patients}

Patients who underwent curative hepatic resection for HCC between January 2009 and May 2015 at West China Hospital were retrospectively reviewed in this study. The following are criteria for the inclusion: (1) pathologically diagnosed as HCC with MVI (2) Child-Pugh A or B liver function; (3) no evidence of extrahepatic metastasis; (4) treated by curative resection, which was defined as no residual tumor and a negative resection (R0) margin based on the histological examination. The exclusion criteria were (1) combined with cholangiocarcinoma or other malignancy; (2) the gross vascular invasion (GVI) located in the main trunk or contralateral branch of portal vein; (3) received other preoperative anticancer treatments; (4) without complete clinical or follow-up data. This study was approved by the Clinical Research Ethics Committee of West China Hospital (IRB number: FWA00009482IRBIORG0004190), and owing to the type of study, formal consents were unnecessary.

\section{Diagnosis and surgery}

Before surgery, each candidate for liver resection had to undergo routine blood tests, conventional coagulation tests, liver and renal function tests, HBV/HCV screening tests, HBV deoxyribonucleic acid (HBV-DNA) measurements, and the examination of serum tumor markers including alpha-fetal protein (AFP), carcinoembryonic antigen, carbohydrate antigen 19-9 (CA199) and CA-125. Electrocardiogram and chest radiography were performed routinely, pulmonary function test and echocardiography were regularly carried out for patients with age more than 65 years old or when clinically necessary. Three-phaseenhanced computed tomography (CT) or magnetic resonance imaging (MRI) scans were performed to preliminarily evaluate the characteristics of the tumors and plan surgery strategy.

The surgical procedures were determined preoperatively based on tumor status, liver function, possibility of adequate surgical margin and technical difficulty in liver resection. Anatomic resection was the preferred option for each patient. During surgery, intraoperative ultrasonography was regularly applied to guide the resection margin and identify additional nodules missed by preoperative imaging., ${ }^{20,21}$ The two most commonly used methods for parenchymal dissection were Cavitron Ultrasonic Surgical Aspirator and clamp crushing. We employed hemihepatic vascular inflow occlusion or the intermittent Pringle's maneuver to control surgical blood loss. Anesthesia was maintained by reducing the tidal volume of ventilation to $30 \%$ to $40 \%$ of the standard volume and keeping the central venous pressure below $5 \mathrm{~cm} \mathrm{H}_{2} \mathrm{O}$ to decrease the amount of blood loss from the hepatic veins during hepatectomy.

\section{Pathological examination}

The resected specimens were embedded in paraffin and delivered to histological department for histological examination. The differentiation grade was confirmed based on the criteria of the Edmondson-Steiner classification. ${ }^{22}$ Satellite lesions were defined as separate nodules within $2 \mathrm{~cm}$ both in size and distance from primary tumor. ${ }^{15,23}$ The diagnosis of MVI was based on "the evidence-based practice guidelines for standardized pathological diagnosis of primary liver cancer in China: 2015 update,"23 which was commonly used in clinical practice in China and cited by multiple previous literatures. ${ }^{10,24}$ The histological diagnosis of each patient was performed by two professional pathologists; any disagreement was resolved by a group discussion.

\section{Follow-up}

After discharge, all patients were regularly followed up at the first postoperative month and then at 3-month intervals in the first 3 years and every 6 months thereafter. Laboratory examinations including routine blood tests, liver function tests, AFP and HBV-DNA load, abdominal ultrasonography, and chest Xray were generally carried out. Enhanced abdominal CT/MRI was performed when suspicious lesions were found or AFP showed persistent elevation. Bone scintigraphy was applied to confirm the bone metastases. For patients with HBV-DNA level more than $10^{3}$ copies $/ \mathrm{mL}$, the usage of antiviral drugs with a low rate of resistance (e.g., entecavir or tenofovir disoproxil) were monitored. ${ }^{25}$ Once the recurrence was confirmed, appropriate therapies including liver resection, radiofrequency ablation 
(RFA), liver transplantation, transarterial chemoembolization (TACE), sorafenib and/or best care protocol were recommended accordingly. The endpoints of this study were the 1, 2 and 3-year disease-free survival (DFS) and overall survival (OS) rate. We defined the DFS as the interval between the date of liver resection and the date of confirmation of recurrence. The OS was the interval between the date of surgery and the date of death or the last follow-up.

\section{Statistical analysis}

All statistical analyses were conducted using SPSS version 21.0 (IBM Corp., Armonk, NY, USA), Prism version 5.0 (GraphPad Software, San Jose, CA, USA), and R software version 3.5.0 with the rms package (The R Foundation, Vienna, Austria; http://www.r-project.org/). Categorical variables were expressed as count or percent and compared using the chisquare or Fisher exact test as appropriate. Continuous data were reported as mean \pm standard deviation and were compared using the Student t-test, one-way analysis of variance or MannWhitney test as appropriate. The survival curves were plotted using the Kaplan-Meier method and compared using the logrank test. All included patients were randomly divided into training and validation cohorts in the ratio of 2 to 1 , which were used to construct and validate the nomograms respectively. Univariate analysis was carried out to investigate potential risk factors, then the factors with a p-value less than 0.05 in the univariate analysis were entered into stepwise multivariate analysis using Cox model to identify independent risk factors of the DFS and OS, respectively. The establishment of nomograms was based on the results of independent risk factors derived from multivariate analysis in training cohort. The predictive accuracy of nomograms was evaluated by the Harrell's C-index; a bootstrap with 1,000 resamples was performed to reduce biased estimate. A larger $\mathrm{C}$-index value represents more accurate prediction. The differences of the C-index between the nomogram and other staging systems were analyzed using the rcorrp.cens package in $\mathrm{R}^{19}$ The calibration curves were applied to illustrate the agreement between the nomogram-predicted and actual observed Kaplan-Meier estimates for survival probability. External validations of nomograms were carried out in validation cohort using the same methods. The discrimination ability of nomograms was estimated by stratifying the prognostic outcomes of patients using the risk scores generated from the nomograms. All statistical tests were two-tailed, p-value less than 0.05 was considered statistically significant.

\section{RESULTS}

\section{Clinical characteristics and prognostic outcomes of all included patients}

From January 1, 2009 to May 31, 2015, a total of 385 consecutive patients met our eligible criteria were enrolled in this study. Then 255 and 130 patients were randomly assigned to the training and validation cohorts, respectively. The detailed clinicopathological information of patients in different cohorts are listed in Table 1. In brief, most patients were male (male/female: 339/46), the average age of patients was $50.1 \pm 12.5$ years ranging from 16 to 80 years. The hepatitis B surface antigen (HBsAg) was detected in 342 patients (88.83\%), and 71 patients (20.0\%) were hepatitis B e antigen (HBeAg) positive. One hundred seventy-eight patients (46.23\%) had HBV-DNA load more than $10^{3}$ copies/mL, and 56 out of 178 (31.46\%) were treatmentnaïve patients to whom entecavir or tenofovir disoproxil were added before surgery. During follow-up, the usage of antiviral drugs was seriously monitored and the HBV-DNA load was regularly detected for patients with aberrant HBV-DNA load. AFP levels were significantly elevated in 226 patients (57.66\%, $\geq 400 \mathrm{ng} / \mathrm{mL}$ ). The preoperative image findings showed that the average diameter of largest tumor was $8.0 \mathrm{~cm}$ (range, 1.8 to 20 $\mathrm{cm})$, multiple nodules were identified in 101 patients (26.23\%), and 129 patients (33.51\%) had GVI which was defined as the tumor embolus being observed in the first or second branches of the portal veins by preoperative imaging. With respect to the histological results, more than half of the patients (54.81\%) had poor grade of tumor differentiation. Liver cirrhosis were verified in 168 patients (43.64\%) with the Ishak score of 6. The Glisson's capsule invasion which was defined as the tumor cells invading but not penetrating the liver capsule was found in 107 patients (27.29\%), 79 patients (20.52\%) had satellite lesions and 14 patients (3.64\%) had positive hepatic lymph node metastasis. The follow-up was censored in May 31, 2018 with a median followup period of 17 months ranging from 1 to 111 months. During the follow-up period, the majority of the patients $(333 / 385$, 86.49\%) experienced tumor recurrence, and recurrence occurred in 71.7\% within the first postoperative year. Among them, 18 patients (4.67\%) underwent rehepatectomy, 14 (3.63\%) underwent RFA, five patients (1.30\%) received salvage liver transplantation, and 160 patients (41.56\%) underwent TACE. The median number of TACE was 1 with a range from 1 to 10 . The patients had a median DFS of 5 months with DFS rates of 1-year: 29.3\%, 2-year: 20.6\%, 3-year: 16.6\% (Fig. 1A), and a median OS time of 17 months with OS rates of 1-year: 58.7\%, 2-year: 39.8\%, 3-year: 34.4\% (Fig. 1B).

\section{Independent risk factors of DFS and OS in training cohort}

The results of univariate and multivariate analyses for the DFS and OS in the training cohort are presented in Table 2. The univariate analysis revealed that 12 clinical variables including age, HBV-DNA load, AFP level, neutrophil-to-lymphocyte ratio (NLR), platelet-to-lymphocyte ratio (PLR), gamma-glutamyl transpeptidase, tumor size, tumor number, GVI, differentiation, Glisson's capsule invasion and satellite lesions were significantly associated with both the DFS and OS. Meanwhile, we also found that neutrophil, aspartate aminotransferase and intraop- 
Table 1. The Basic Clinical Characteristics of Patients

\begin{tabular}{|c|c|c|c|}
\hline Clinical parameter & Total $(\mathrm{n}=385)$ & Training cohort $(\mathrm{n}=255)$ & Validation cohort $(\mathrm{n}=130)$ \\
\hline Sex, male/female & $339 / 46$ & $222 / 33$ & $117 / 13$ \\
\hline Age, yr & $50.1 \pm 12.5$ & $49.8 \pm 12.4$ & $50.5 \pm 12.7$ \\
\hline Diabetes, yes/no & $18 / 367$ & $10 / 245$ & $8 / 122$ \\
\hline HBsAg, positive/negative & $342 / 43$ & $223 / 32$ & $119 / 11$ \\
\hline HBeAg, positive/negative & $71 / 314$ & $53 / 202$ & $17 / 113$ \\
\hline Anti-HCV, positive/negative & $3 / 382$ & $2 / 253$ & $1 / 129$ \\
\hline HBV-DNA, $>10^{3} /<10^{3}$ copies $/ \mathrm{mL}$ & $178 / 207$ & $117 / 138$ & $61 / 69$ \\
\hline $\mathrm{AFP}, \leq 20 / 20-400 />400 \mathrm{ng} / \mathrm{mL}$ & $85 / 74 / 226$ & $50 / 53 / 152$ & $35 / 21 / 74$ \\
\hline CA19-9, U/mL & $30.5 \pm 57.4$ & $32.3 \pm 54.5$ & $27.1 \pm 62.5$ \\
\hline CA-125, U/mL & $25.9 \pm 31.5$ & $28.3 \pm 36.6$ & $21.6 \pm 18.4$ \\
\hline CEA, ng/mL & $8.74 \pm 25.4$ & $7.41 \pm 15.1$ & $11.3 \pm 38.1$ \\
\hline WBC, $10^{9} / \mathrm{L}$ & $6.0 \pm 2.6$ & $5.9 \pm 2.6$ & $6.1 \pm 2.4$ \\
\hline $\mathrm{NEU}, 10^{9} / \mathrm{L}$ & $4.0 \pm 2.4$ & $3.9 \pm 2.4$ & $4.0 \pm 2.4$ \\
\hline LYM, $10^{9} / \mathrm{L}$ & $1.4 \pm 0.6$ & $1.4 \pm 0.6$ & $1.5 \pm 0.6$ \\
\hline NLR & $3.3 \pm 3.2$ & $3.3 \pm 3.0$ & $3.3 \pm 3.7$ \\
\hline $\mathrm{RBC}, 10^{12} / \mathrm{L}$ & $4.8 \pm 0.8$ & $4.7 \pm 0.8$ & $4.8 \pm 0.8$ \\
\hline HGB, g/L & $142.3 \pm 19.8$ & $141.7 \pm 20.5$ & $143.4 \pm 18.5$ \\
\hline PLT, $10^{9} / \mathrm{L}$ & $155.0 \pm 77.1$ & $152.6 \pm 72.7$ & $159.6 \pm 85.1$ \\
\hline PLR & $127.8 \pm 114.1$ & $125.8 \pm 83.4$ & $131.7 \pm 158.3$ \\
\hline PT, sec & $12.3 \pm 1.24$ & $12.3 \pm 1.2$ & $12.3 \pm 1.3$ \\
\hline INR & $1.1 \pm 0.4$ & $1.1 \pm 0.5$ & $1.1 \pm 0.1$ \\
\hline TBIL, $\mu \mathrm{mol} / \mathrm{L}$ & $20.9 \pm 63.8$ & $17.5 \pm 29.8$ & $27.7 \pm 101.6$ \\
\hline ALB, $\mathrm{g} / \mathrm{L}$ & $41.0 \pm 5.3$ & $41 \pm 5.4$ & $41 \pm 5.0$ \\
\hline AST, IU/L & $65.0 \pm 65.1$ & $62.8 \pm 57.1$ & $69.3 \pm 78.6$ \\
\hline ALT, IU/L & $61.0 \pm 63.1$ & $58.2 \pm 58.6$ & $66.5 \pm 71.0$ \\
\hline GGT, IU/L & $139.5 \pm 141.8$ & $149.1 \pm 152.6$ & $120.5 \pm 116.0$ \\
\hline Child-Pugh, A/B & $361 / 24$ & $239 / 16$ & $122 / 8$ \\
\hline Size, cm & $8.0 \pm 3.7$ & $8.1 / 3.8$ & $7.6 / 3.5$ \\
\hline No., single/multiple & $284 / 101$ & $188 / 67$ & $96 / 34$ \\
\hline GVI, yes/no & $129 / 256$ & $88 / 167$ & $41 / 89$ \\
\hline Tumor capsule, complete/infiltrate & $133 / 252$ & $76 / 179$ & $57 / 73$ \\
\hline BCLC stage, $\mathrm{A} / \mathrm{B} / \mathrm{C}$ & $64 / 197 / 124$ & $39 / 127 / 89$ & $21 / 70 / 39$ \\
\hline Anatomic resection, yes/no & $234 / 151$ & $166 / 89$ & $68 / 62$ \\
\hline Blood loss, $\mathrm{mL}$ & $582.8 \pm 759.4$ & $562.1 \pm 524.4$ & $622.1 \pm 1,065.2$ \\
\hline Transfusion, yes/no & $56 / 329$ & $36 / 219$ & $20 / 110$ \\
\hline Differentiation, I+II/III+IV & $175 / 210$ & $115 / 140$ & $60 / 70$ \\
\hline Glisson's capsule invasion, yes/no & $107 / 278$ & $73 / 182$ & $34 / 96$ \\
\hline Satellite, yes/no & $79 / 306$ & $49 / 206$ & $30 / 100$ \\
\hline Node metastasis, yes/no & $14 / 371$ & $9 / 246$ & $5 / 125$ \\
\hline Cirrhosis, yes/no & $168 / 217$ & $124 / 131$ & $45 / 85$ \\
\hline
\end{tabular}

HBsAg, hepatitis B surface antigen; HBeAg, hepatitis B e antigen; Anti-HCV, hepatitis C virus antibody; HBV-DNA, hepatitis B virus deoxyribonucleic acid; AFP, alpha-fetoprotein; CA19-9, carbohydrate antigen 19-9; CA-125, carbohydrate antigen 125; CEA, carcinoembryonic antigen; WBC, white blood cell; NEU, neutrophil; LYM, lymphocyte; NLR, neutrophil-lymphocyte ratio; RBC, red blood cell; HGB, hemoglobin; PLT, platelet; PLR, platelet-to-lymphocyte ratio; PT, prothrombin time; INR, international normalized ratio; TBIL, total bilirubin; ALB, albumin; AST, aspartate aminotransferase; ALT, alanine transaminase; GGT, gamma-glutamyl transpeptidase; GVI, gross vascular invasion; BCLC, Barcelona Clinic Liver Cancer staging system. 
erative blood loss were additional risk factors for the OS. These variables were integrated into stepwise multivariate analyses to identify independent risk factors of the DFS and OS. The results showed that five factors including age (DFS: hazard ratio [HR], 0.98; 95\% confidence interval [CI], 0.97 to 0.99 ; $p=0.002$; 0S: HR, 0.98; 95\% CI, 0.97 to 0.99; $p=0.011$ ), tumor size (DFS: HR, 1.11 ; 95\% CI, 1.06 to 1.15 ; p<0.001; OS: HR, 1.10; 95\% CI, 1.05 to $1.15 ; \mathrm{p}<0.001$ ), tumor number (DFS: HR, 1.50; 95\% CI, 1.08 to 2.08 ; $p=0.015$; OS: HR, $1.49 ; 95 \%$ CI, 1.05 to $2.10 ; p=0.024$ ), GVI (DFS: HR, 1.56 ; 95\% CI, 1.15 to $2.11 ; \mathrm{p}=0.004$; OS: HR, 1.56; 95\% CI, 1.12 to $2.17 ; \mathrm{p}=0.009$ ) and Glisson's capsule invasion (DFS: HR, 1.80 ; 95\% CI, 1.28 to 2.54; $\mathrm{p}=0.001$; OS: $\mathrm{HR}, 1.76$; $95 \% \mathrm{CI}, 1.19$ to $2.60 ; \mathrm{p}=0.005)$ were the independent prognostic factors of both the DFS and OS. And the PLR was an additional independent risk factors for the OS (HR, 1.02; 95\% CI, 1.01 to $1.03 ; \mathrm{p}=0.041$ ).

\section{Construction and validation of the nomograms for DFS and $0 S$}

The nomograms used to predict the DFS and OS were constructed based on above mentioned independent risk factors. For the DFS (Fig. 2A), the elements of nomogram contained two continuous variables (age and tumor size) and three categorical variables (GVI, Glisson's capsule invasion, and tumor number). Younger age, larger tumor size, multiple tumor numbers, presence of GVI or Glisson's capsule invasion were considered as risk factors of lower DFS rate. The bootstrap-corrected C-index of DFS nomograms in training and validation cohorts were 0.712 (95\% CI, 0.679 to $0.745 ; \mathrm{p}<0.001$ ) and 0.704 (95\% CI, 0.650 to $0.708 ; \mathrm{p}<0.001$ ) respectively. With regard to OS (Fig. 2B), the nomogram contained three continuous variables (tumor size, age, and PLR) and three categorical variables (GVI, Glisson's capsule invasion, and tumor number). The bootstrap-corrected C-index of OS nomograms in training and validation cohorts were 0.698 (95\% CI, 0.657 to $0.739 ; \mathrm{p}<0.001)$ and $0.673(95 \%$

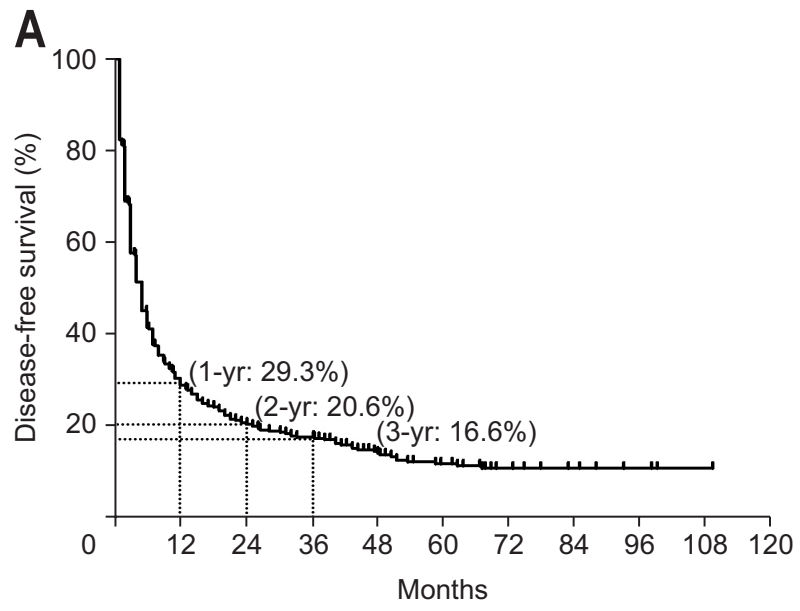

CI, 0.607 to $0.739 ; \mathrm{p}<0.001)$ respectively. The calibration curves of both training and validation cohorts showed optimal agreement between the actual observation and nomogram-predicted probability of DFS (Fig. 3A-F) and OS (Fig. 3G-L) at 1, 2 and 3 -year after surgery. These results suggested that these nomograms had satisfactory application value for prognostic prediction of the HCC patients with MVI after liver resection.

For clinical use of these models, the number of points for each factor was determined by drawing a vertical line toward to the point scale. For the continuous variables (age and tumor size), the number of points was equal to the nearest integer value on the point scale. Then each patient received unique DFS and OS scores by adding the points for all variables in predicted models. The projection of the total points on the survival axes indicated the estimated probability of DFS and OS at 1, 2 and 3 years, respectively.

\section{Comparison of the performance between the novel no- mograms and conventional staging systems}

In this study, the predictive accuracy of six conventional stage systems (Barcelona Clinic Liver Cancer [BCLC], American Joint Committee on Cancer [AJCC], Cancer of the Liver Italian Program [CLIP], Japan Integrated Staging Score [JIS], Okuda, and Hong Kong Liver Cancer prognostic classification scheme [HKLC]) were also calculated (Table 3). Overall, most of them had acceptable ability in predicting the prognosis of HCC patients with MVI with the p-value less than 0.05. However, the C-indices were significantly lower than those of present nomograms. In the training cohort, the C-indices of the nomograms for the DFS and OS were 0.712 and 0.698 respectively, which were significantly higher than those of the BCLC (DFS: 0.595, p<0.001; 0S: 0.589, p<0.001), AJCC seventh (DFS: 0.599, p<0.001; OS: 0.590, $p<0.001$ ), CLIP (DFS: 0.653, $p=0.005$; 0S: 0.655, $\mathrm{p}=0.047$ ), JIS (DFS: 0.588, $\mathrm{p}<0.001$; OS: 0.590, $\mathrm{p}<0.001$ ), Okuda (DFS: $0.564, \mathrm{p}<0.001$; OS: $0.578, \mathrm{p}<0.001$ ) and HKLC

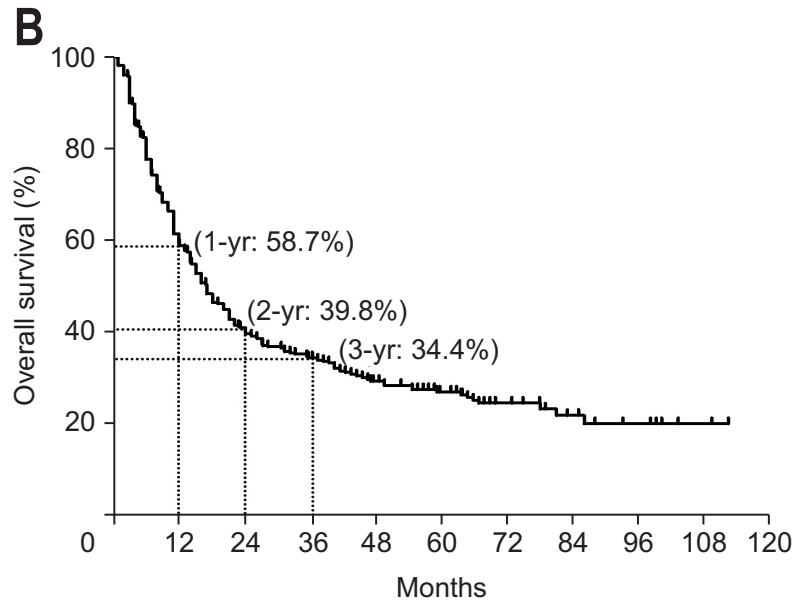

Fig. 1. Kaplan-Meier estimates of the prognosis of hepatocellular carcinoma patients with microvascular invasion. (A) Disease-free survival. (B) Overall survival. 
Table 2. Prognostic Factors Correlated with DFS and OS Based on the Cox Proportional Hazards Model

\begin{tabular}{|c|c|c|c|c|}
\hline \multirow{2}{*}{ Clinical parameter } & \multicolumn{2}{|c|}{ DFS } & \multicolumn{2}{|c|}{ OS } \\
\hline & HR (95\% CI) & p-value & $\mathrm{HR}(95 \% \mathrm{CI})$ & p-value \\
\hline \multicolumn{5}{|l|}{ Univariate analysis } \\
\hline Sex, male/female & $0.96(0.61-1.50)$ & 0.85 & $1.20(0.73-1.99)$ & 0.476 \\
\hline Age & $0.68(0.51-0.92)$ & 0.011 & $0.62(0.45-0.85)$ & 0.003 \\
\hline HBsAg, positive/negative & $1.08(0.70-1.67)$ & 0.74 & $1.29(0.78-2.14)$ & 0.32 \\
\hline HBeAg, positive/negative & $1.17(0.82-1.65)$ & 0.387 & $1.34(0.92-1.95)$ & 0.123 \\
\hline Anti-HCV, positive/negative & $0.30(0.04-2.17)$ & 0.235 & $0.48(0.07-3.44)$ & 0.466 \\
\hline HBV-DNA, $\geq 10^{3} /<10^{3}$ copies $/ \mathrm{mL}$ & $1.42(1.03-1.96)$ & 0.035 & $1.87(1.31-2.67)$ & 0.001 \\
\hline $\mathrm{AFP}, \leq 400 />400 \mathrm{ng} / \mathrm{mL}$ & $1.52(1.12-2.07)$ & 0.007 & $1.41(1.14-1.74)$ & 0.002 \\
\hline CA19-9 & $0.95(0.69-1.29)$ & 0.738 & $1.10(0.79-1.54)$ & 0.567 \\
\hline CA-125 & $1.57(0.99-2.49)$ & 0.058 & $1.53(0.94-2.50)$ & 0.09 \\
\hline CEA & $1.06(0.76-1.48)$ & 0.743 & $1.25(0.87-1.79)$ & 0.235 \\
\hline WBC & $1.37(0.93-2.03)$ & 0.11 & $1.33(0.87-2.04)$ & 0.181 \\
\hline NEU & $1.32(0.91-1.91)$ & 0.142 & $1.86(1.20-2.89)$ & 0.005 \\
\hline LYM & $0.91(0.67-1.24)$ & 0.554 & $0.87(0.63-1.22)$ & 0.419 \\
\hline $\mathrm{NLR}, \leq 3 />3$ & $1.54(1.14-2.08)$ & 0.005 & $1.86(1.35-2.58)$ & $<0.001$ \\
\hline $\mathrm{RBC}$ & $0.88(0.63-1.23)$ & 0.454 & $0.90(0.62-1.29)$ & 0.559 \\
\hline HGB & $0.88(0.61-1.26)$ & 0.49 & $0.73(0.50-1.07)$ & 0.104 \\
\hline PLT & $1.38(0.99-1.94)$ & 0.061 & $1.39(0.96-2.01)$ & 0.08 \\
\hline $\mathrm{PLR}, \leq 111 />111$ & $1.66(1.23-2.23)$ & 0.001 & $1.74(1.26-2.39)$ & 0.001 \\
\hline PT & $0.94(0.68-1.29)$ & 0.684 & $0.94(0.67-1.33)$ & 0.734 \\
\hline INR & $0.97(0.70-1.35)$ & 0.856 & $1.01(0.70-1.46)$ & 0.963 \\
\hline TBIL & $0.55(0.26-1.17)$ & 0.118 & $0.83(0.39-1.78)$ & 0.632 \\
\hline ALB & $0.87(0.64-1.16)$ & 0.339 & $0.76(0.55-1.04)$ & 0.087 \\
\hline AST & $1.24(0.91-1.69)$ & 0.168 & $1.60(1.13-2.24)$ & 0.008 \\
\hline ALT & $1.05(0.78-1.41)$ & 0.736 & $1.21(0.88-1.66)$ & 0.25 \\
\hline GGT & $1.67(1.18-2.35)$ & 0.003 & $2.09(1.42-3.10)$ & $<0.001$ \\
\hline Child-Pugh, A/B & $1.01(0.58-1.75)$ & 0.971 & $1.06(0.62-1.83)$ & 0.821 \\
\hline Tumor size & $1.57(1.26-1.96)$ & $<0.001$ & $1.12(1.08-1.17)$ & $<0.001$ \\
\hline Tumor number, single/multiple & $1.50(1.08-2.08)$ & 0.015 & $1.47(1.04-2.07)$ & 0.028 \\
\hline Tumor capsule, complete/infiltrate & $0.77(0.50-1.18)$ & 0.225 & $0.79(0.49-1.27)$ & 0.335 \\
\hline GVI, yes/no & $1.71(1.27-2.32)$ & $<0.001$ & $1.78(1.28-2.46)$ & 0.001 \\
\hline BCLC stage, $\mathrm{A} / \mathrm{B} / \mathrm{C}$ & $1.50(1.19-1.88)$ & $<0.001$ & $1.57(1.22-2.01)$ & $<0.001$ \\
\hline Anatomic resection, yes/no & $1.07(0.79-1.46)$ & 0.652 & $1.37(0.97-1.94)$ & 0.079 \\
\hline Blood loss & $1.39(0.98-1.97)$ & 0.064 & $1.79(1.22-2.63)$ & 0.003 \\
\hline Transfusion, yes/no & $1.20(0.77-1.86)$ & 0.424 & $1.37(0.87-2.16)$ & 0.174 \\
\hline Differentiation, I+II/III+IV & $1.40(1.04-1.89)$ & 0.025 & $1.68(1.21-2.32)$ & 0.002 \\
\hline Liver capsule invasion, yes/no & $1.75(1.24-2.46)$ & 0.001 & $1.93(1.31-2.85)$ & 0.001 \\
\hline Satellite, yes/no & $1.60(1.12-2.29)$ & 0.011 & $1.59(1.09-2.31)$ & 0.016 \\
\hline Node metastasis, yes/no & $0.92(0.38-2.23)$ & 0.847 & $1.43(0.58-3.48)$ & 0.435 \\
\hline Cirrhosis, yes/no & $1.11(0.80-1.54)$ & 0.526 & $1.35(0.95-1.93)$ & 0.099 \\
\hline \multicolumn{5}{|l|}{ Multivariate analysis } \\
\hline Age & $0.98(0.97-0.99)$ & 0.002 & $0.98(0.97-0.99)$ & 0.011 \\
\hline Tumor size & $1.11(1.06-1.15)$ & $<0.001$ & $1.10(1.05-1.15)$ & $<0.001$ \\
\hline Tumor number, single/multiple & $1.50(1.08-2.08)$ & 0.015 & $1.49(1.05-2.10)$ & 0.024 \\
\hline GVI, yes/no & $1.56(1.15-2.11)$ & 0.004 & $1.56(1.12-2.17)$ & 0.009 \\
\hline Glisson's capsule invasion, yes/no & $1.80(1.28-2.54)$ & 0.001 & $1.76(1.19-2.60)$ & 0.005 \\
\hline PLR & - & - & $1.02(1.01-1.03)$ & 0.041 \\
\hline
\end{tabular}

DFS, disease-free survival; OS, overall survival; HR, hazard ratio; CI, confidence interval; HBsAg, hepatitis B surface antigen; HBeAg, hepatitis B e antigen; Anti-HCV, hepatitis C virus antibody; HBV-DNA, hepatitis B virus deoxyribonucleic acid; AFP, alpha-fetoprotein; CA19-9, carbohydrate antigen 19-9; CA-125, carbohydrate antigen 125; CEA, carcinoembryonic antigen; WBC, white blood cell; NEU, neutrophil; LYM, lymphocyte; NLR, neutrophil-to-lymphocyte ratio; RBC, red blood cell; HGB, hemoglobin; PLT, platelet; PLR, platelet-to-lymphocyte ratio; PT, prothrombin time; INR, international normalized ratio; TBIL, total bilirubin; ALB, albumin; AST, aspartate aminotransferase; ALT, alanine transaminase; GGT, gamma-glutamyl transpeptidase; GVI, gross vascular invasion; BCLC, Barcelona Clinic Liver Cancer staging system. 
A

Points

Tumor size $(\mathrm{cm})$

GVI

Glisson's capsule invasion

Age (yr)

Tumor number

Total points

1-yr DFS

2-yr DFS

3-yr DFS

B

Points

Tumor size $(\mathrm{cm})$

GVI

Glisson's capsule invasion

Age (yr)

Tumor number

PLR

Total points

$1-\mathrm{yr}$ OS

2-yr OS

3-yr OS
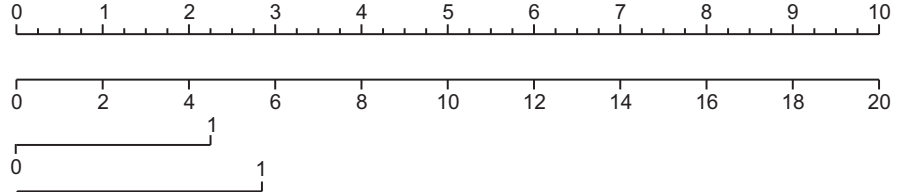

0

$\begin{array}{llllllllllllllll}85 & 10 & 1 & 1 & 1 & 1 & 1 & 1 & 1 & 1 & 1 & 1 & 1 & 1 & 1 & 1\end{array}$ 1

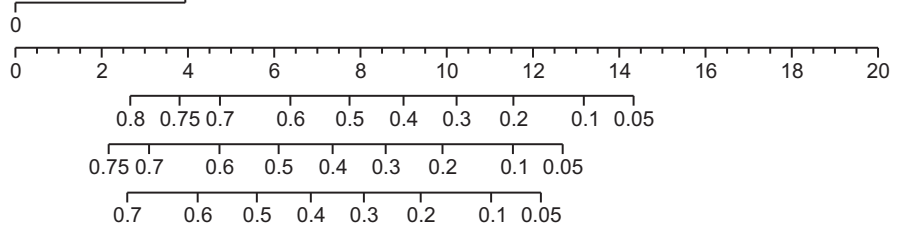

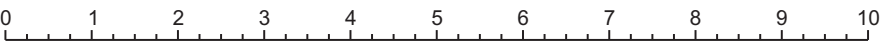

\begin{tabular}{ll|llllllllll}
\hline 0 & 2 & 4 & & 6 & 8 & 10 & 12 & 14 & 16 & 18 & 20
\end{tabular}

\begin{tabular}{lllllllllllllllllll}
\hline 85 & 1 & 1 & 1 & 1 & 1 & 1 & 1 & 1 & 1 & 1 & 1 & 1 & 1 & \\
8 & 80 & 75 & 70 & 65 & 60 & 55 & 50 & 45 & 40 & 35 & 30 & 25 & 20 & 15
\end{tabular}

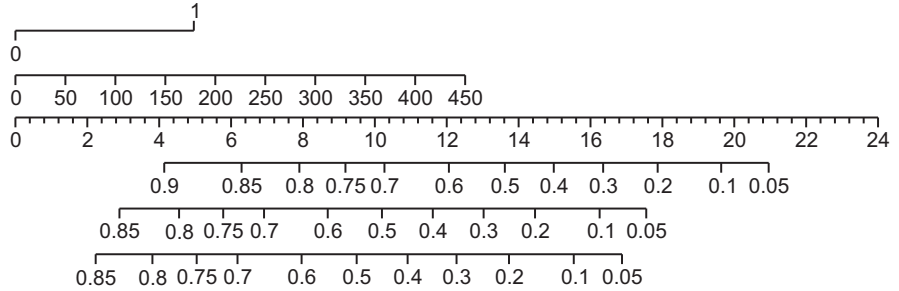

Fig. 2. Nomograms for predicting disease-free survival (DFS) and overall survival (OS) in hepatocellular carcinoma patients with microvascular invasion. (A) DFS. (B) OS. GVI, gross vascular invasion; PLR, platelet-to-lymphocyte ratio.
(DFS: 0.599, $\mathrm{p}<0.001$; 0S: $0.595, \mathrm{p}<0.001)$. In the validation cohort, the C-indices of the nomograms for the DFS and OS were 0.704 and 0.673 respectively, which were also significantly higher than those of the BCLC (DFS: 0.610, p<0.001; 0S: 0.585, p<0.001), AJCC seventh (DFS: 0.604, p<0.001; 0S: 0.565, $\mathrm{p}<0.001$ ), CLIP (DFS: 0.620, $p=0.02$; OS: 0.618, $p=0.025$ ), JIS (DFS: 0.604, p<0.001; 0S: 0.579, p<0.001), Okuda (DFS: 0.564, $\mathrm{p}<0.001$; OS: $0.549, \mathrm{p}<0.001$ ), and HKLC (DFS: 0.623, $\mathrm{p}<0.001$; OS: 0.613, $\mathrm{p}<0.001)$.

\section{Comparison of the discrimination ability between the novel nomograms and conventional staging systems}

Using above two nomograms, the total number of points of each patient was calculated. For DFS, the total number of points of each patient ranged from 4 to 19 . Then all patients were divided into four subgroups based on an interval of number of points of 4 (4-7, 8-11, 12-15, and 16-19). As shown in Fig. 4A, each risk group had a distinct rate of DFS $(\mathrm{p}<0.001)$. In terms of OS, the total number of points of each patient ranged from 5 to 23. Similarly, all patients were stratified into four subgroups based on an interval of number of points of 5 (5-9, 10-14, 15-19, and 20-23), each risk group also had a distinct rate of OS $(\mathrm{p}<0.001)$ (Fig. 4B).
Additionally, the survival curves of DFS and OS for all eligible patients were depicted by six widely used staging systems of HCC (BCLC, AJCC, CLIP, JIS, Okuda, and HKLC) (Fig. 5). Compared with the survival curves derived from present nomograms, only Okuda system could stratify patients into two risk groups (Fig. 5E, H); BCLC (Fig. 5A, G) and HKLC (Fig. 5F, L) systems were unsatisfactory in stratifying patients between stages II and III for DFS; AJCC seventh (Fig. 5B, H) was unsatisfactory in stratifying patients over stage II; the CLIP score system (Fig. 5C, I) was unsatisfactory in stratifying patients between 0 and 1 as well as between 3 and 4; JIS score system (Fig. 5D, J) was unsatisfactory in stratifying patients between 0 and 1 as well as between 3 and 4. Taken together, the present novel nomograms had more satisfactory discrimination ability than conventional staging systems.

\section{DISCUSSION}

To the best of our knowledge, we reported the largest sample size of HCC patients with MVI. The participants in the present study were distributed in different disease stages and had heterogeneous clinical characteristics, so satisfactory representative of this cohort was considered. Meanwhile, approximately 40 
A Training set

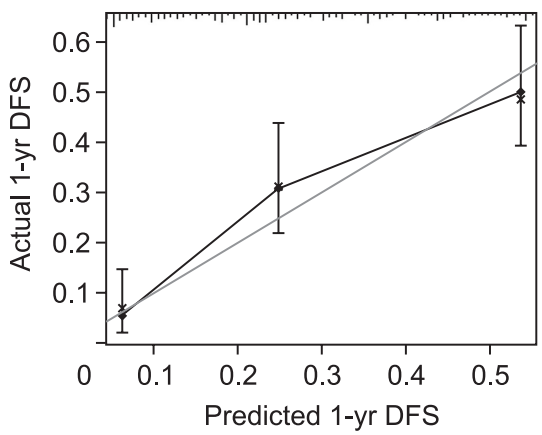

Validation set

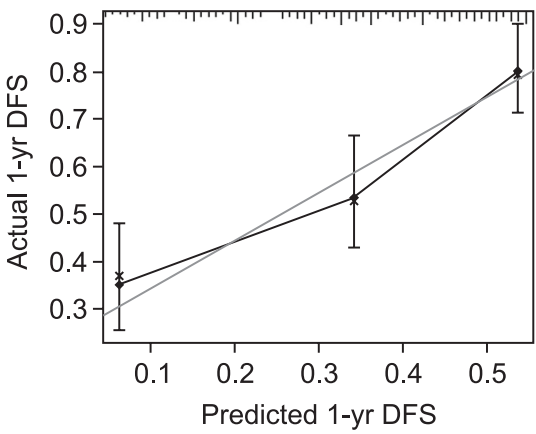

G Training set

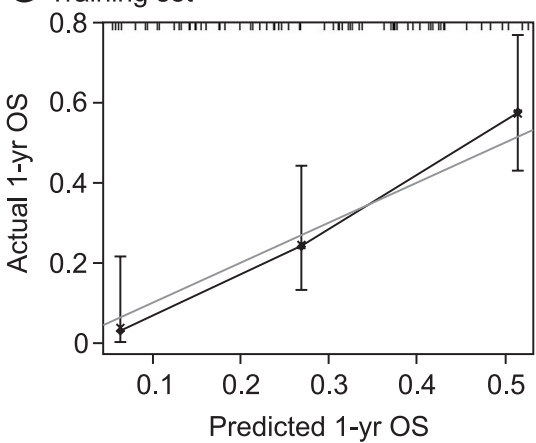

J Validation set

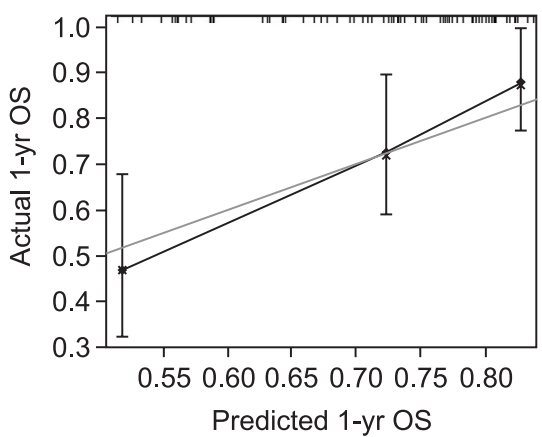

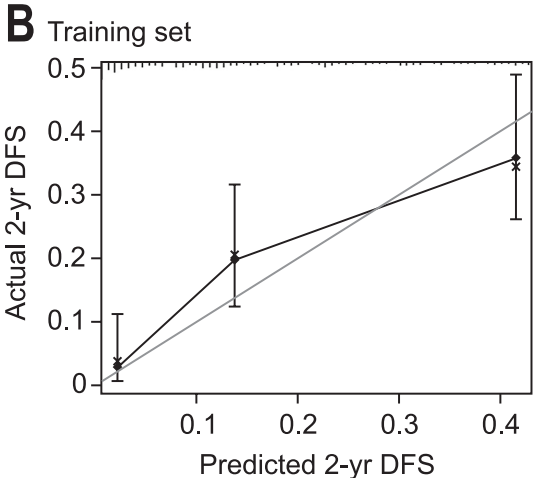

E Validation set

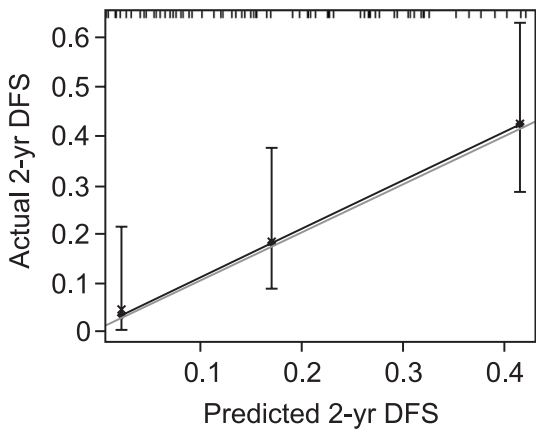

H Training set

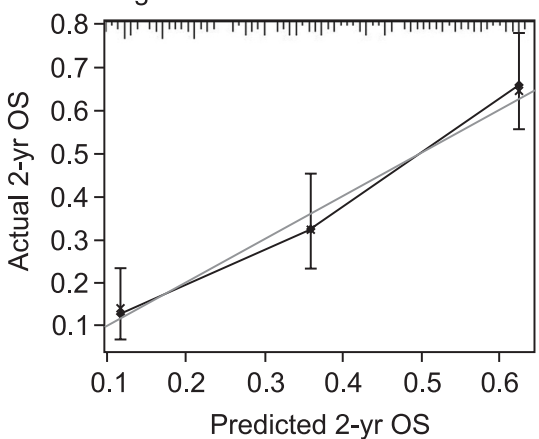

$\mathrm{K}$ Validation set

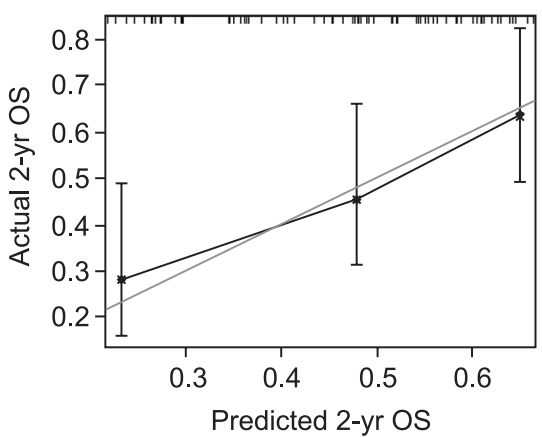

C Training set

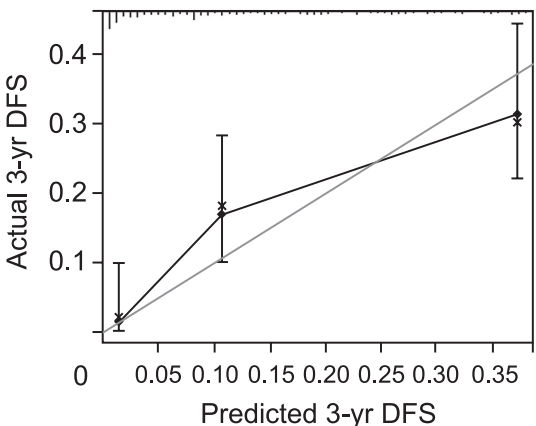

F Validation set

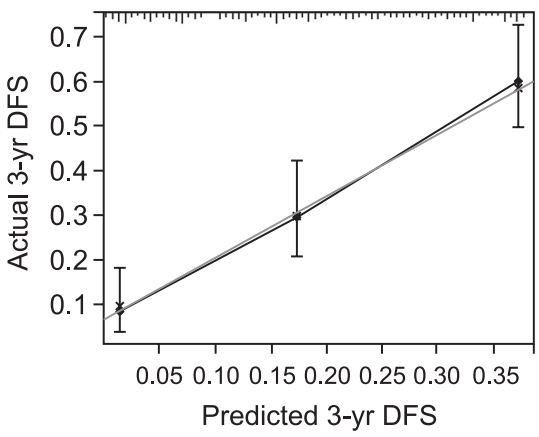

I Training set

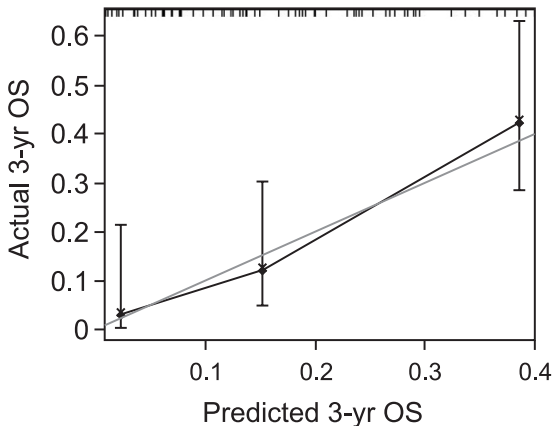

L Validation set

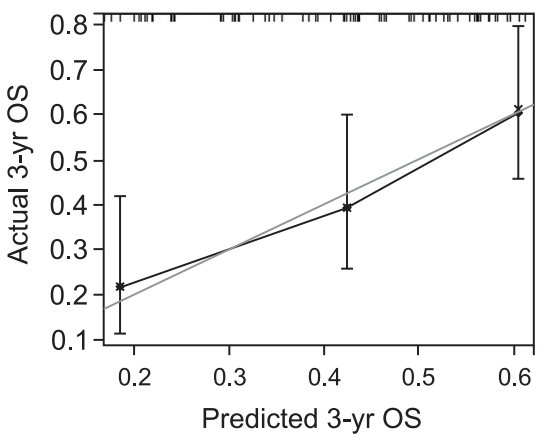

Fig. 3. Calibration curves for predicting disease-free survival (DFS) and overall survival (OS) using the nomograms. (A-C) 1, 2, and 3-year DFS in the training cohort; (D-F) 1, 2, and 3-year DFS in the validation cohort; (G-I) 1, 2, and 3-year OS in the training cohort; (J-L) 1, 2, and 3-year OS in the validation cohort.

clinical parameters of each candidate were collected, which was expected to identify more prognosis-related variables. Via univariate and multivariate analyses, five DFS related and six OS related risk factors were finally identified. Then these independent factors were integrated and two prognosis nomograms for DFS and OS were constructed. Compared with six conventional 


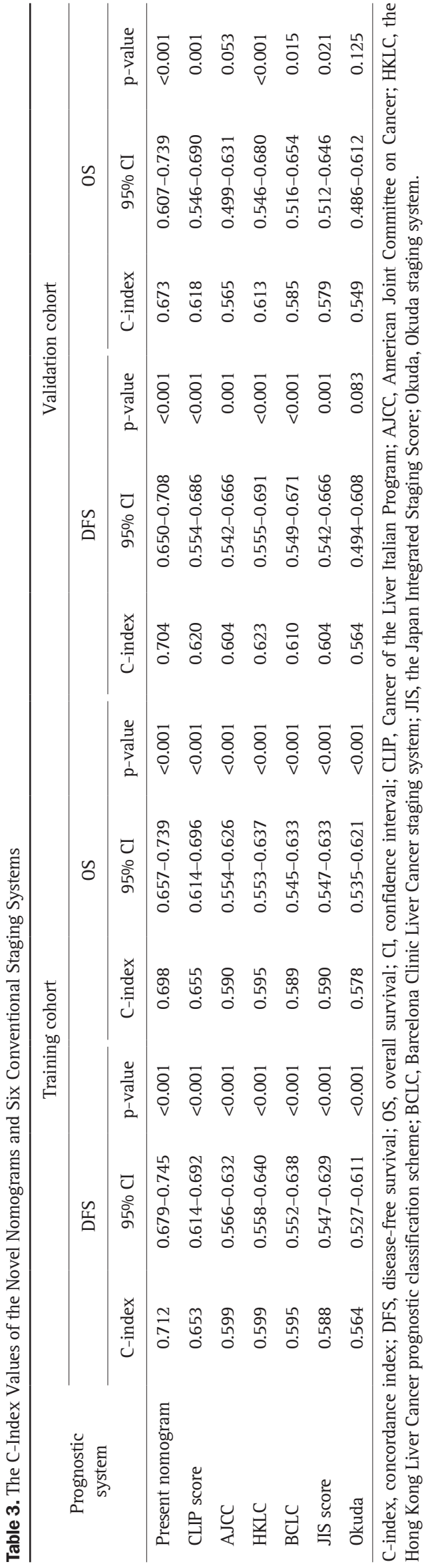

staging systems, these novel predictive models had significantly higher prediction accuracy with C-indices of 0.712 and 0.698 for DFS and OS in training cohort respectively, and 0.704 and 0.673 for DFS and OS in validation cohort respectively. Furthermore, the Kaplan-Meier curves also showed these novel nomograms had more satisfactory discrimination ability than conventional staging systems for stratifying the prognosis of HCC patients with MVI. These results indicated that the present nomograms were convenient and powerful for predicting the prognostic outcomes of patients with MVI, and they may be helpful for guiding surgeons to plan personalized surveillance strategies and design adjuvant therapies after surgery for this subset of HCC patients.

Compared with multiple conventional staging systems, besides tumor size, ${ }^{26-30}$ tumor number ${ }^{28,30-32}$ and $\mathrm{GVI}^{28,29,33}$ which were well-known risk factors of HCC, some novel risk factors including age, and Glissons's capsule invasion were identified to be associated with both the DFS and OS of HCC patients with MVI, and PLR was an additional risk factor for OS in this study. To quantify the risk level of age and PLR more precisely, we expressed these two parameters as continuous variables. As shown in Fig. 2, the risk points were gradually elevated with age. This results were consistent with some previous studies. ${ }^{34-37}$ They found that elderly patients normally had significantly lower positivity rate of HBsAg, lower level of AFP and less advanced tumor characteristics when compared with younger patients. Therefore, a comparable or even longer median survival time was observed in elderly patients. Furthermore, the underlying mechanism on the correlation between the age and the carcinogenesis and progression of HCC were also investigated by previous studies. ${ }^{38,39}$ A gene analysis which was conducted by Yan et al. ${ }^{39}$ compared the genotype and integration patterns of $\mathrm{HBV}$ in early- and late-onset HCC. This novel study revealed that HBV B2 serving as a risk factor of early-onset HCC is predominantly present in younger HCC patients. The HBV integration into 8q24 frequently occurs in younger patients, which effectively induced the over-expression of three well-demonstrated oncogenes (cMYC, PVT1, and microRNA-1204) ${ }^{40-42}$ in HCC tumor tissues.

Glisson's capsule is defined as the connective collagenous layer surrounding the liver parenchyma. ${ }^{43}$ Glisson's capsule invasion is considered that the tumor cells invaded into but not penetrated the Glisson's capsule on microscopy. Previous studies have reported that the elasticity, thickness and texture of Glisson's capsule were associated with the stage of liver fibrosis. ${ }^{43,44}$ However, to our knowledge, the clinical significance of Glisson's capsule invasion in predicting the prognosis of HCC have not been reported. In this study, we identified that Glisson's capsule invasion is a novel independent risk factor of both DFS (HR, 1.80 ; 95\% CI, 1.28 to $2.54 ; \mathrm{p}=0.001$ ) and OS (HR, 1.76; 95\% CI, 1.19 to $2.60 ; \mathrm{p}=0.005)$. In the training cohort, Glisson's capsule invasion promoted the C-indices of DFS from 0.695 to 0.712 , and promoted the C-indices of OS from 0.688 to 0.698 . And 

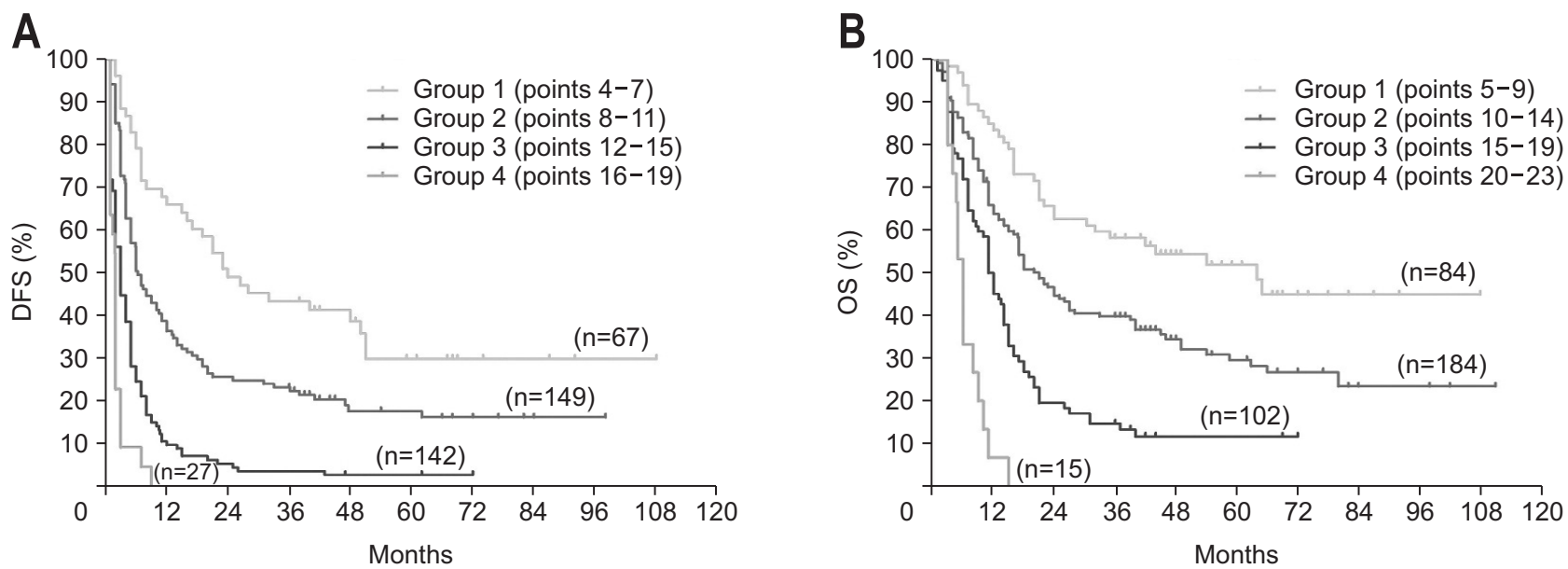

Fig. 4. Kaplan-Meier estimates of the prognosis of patients according to the number of points generated using the nomograms. (A) The rates of disease-free survival (DFS) in the four risk groups were significantly different. (B) The rates of overall survival (OS) in the four risk groups were significantly different. Note: The total number of points for each patient was calculated from the nomogram plots in Fig. 2. Then, the patients were divided into four risk subgroups based on 4-point (DFS) or 5-point (OS) intervals.

in the validation cohort, Glisson's capsule invasion promoted the C-indices of DFS from 0.672 to 0.704 , and promoted the Cindices of OS from 0.662 to 0.673 . Actually, the clinical value of organ capsule invasion has been identified and incorporated into the TNM staging system in multiple solid tumors. In thymoma, tumor invading the capsule was treated as an essential evidence for advanced stage ${ }^{45}$ and an indicator of long term surveillance. ${ }^{46}$ In thyroid cancer, the microscopic invasion of the thyroid capsule was defined as tumor penetration into the thyroid capsule without attachment to the surrounding muscular components, ${ }^{47,48}$ which was associated with poor tumor size, frequent vascular invasion ${ }^{49}$ and unfavorable prognosis. ${ }^{50,51}$ In lung cancer, visceral pleural invasion was an independent risk factor of prognosis in each stage of TNM system. ${ }^{52,53}$ Therefore, the clinical significance of Glisson's capsule invasion in HCC patients without MVI and corresponding mechanisms need to be further investigated.

Recently, accumulating evidence have demonstrated that the aberrantly expressed inflammatory biomarkers prior to surgery such as PLR, NLR, C-reactive protein were prognostic factors in various solid tumors. ${ }^{54-58}$ In this study, we also identified that PLR is independently associated with the OS of patients with MVI (HR, 1.02; 95\% CI, 1.01 to $1.03 ; \mathrm{p}=0.041$ ). Multiple studies have investigated the underlying mechanisms of PLR in the recurrence and metastasis of HCC. The relatively overexpressed platelets produced high levels of multiple tumor-related molecules such as vascular endothelial growth factor, platelet-derived growth factor and platelet-derived serotonin, ${ }^{59-61}$ which were closely correlated with the proliferation, migration and angiogenesis of HCC in vivo and in vitro. Furthermore, platelets could protect free tumor cells from immune system in the circulating system, resulting in higher possibility of intra- and/or extrahepatic metastasis. ${ }^{62}$ Additionally, the patients with high levels of
PLR normally accompanied with lower level of lymphocytes. Investigators have reported that low level of lymphocytic infiltration in tumor tissues was a poor prognostic factor of colorectal and HCC. ${ }^{63}$

Some limitations of this study need to be noted. Firstly, future external validations are necessary as the results of this study were calculated using the data from a single institution. Secondly, although the predicted models did not include virusrelated variables such as HBV-DNA load and HBeAg, its application value for other etiologies related to HCCs needs to be further validated owing to the fact that patients in this study had underlying HBV infections. Thirdly, these nomograms only could be used to estimate the prognosis of patients with MVI after surgery; it is necessary to combine with preoperative MVI prediction models ${ }^{11,16}$ to manage this subset of patients as good as possible. Finally, as these nomograms were established only based on the clinical factors, other prognosis-related biomarkers need to be identified and incorporated to further promote the accuracy of these nomograms.

In conclusion, this study identified younger age, larger tumor size, multiple tumor number, GVI and Glisson's capsule invasion were the independent prognostic factors of both the DFS and OS for HCC patients with MVI following liver resection. Higher PLR was another risk factor of the OS. Two novel predictive nomograms based on these risk factors achieved more satisfactory accuracy and discrimination ability than conventional staging systems. These nomograms may be valuable in determination of individualized surveillance and adjuvant therapy for HCC patients with MVI after surgery.

\section{CONFLICTS OF INTEREST}

No potential conflict of interest relevant to this article was reported. 
A bCLC

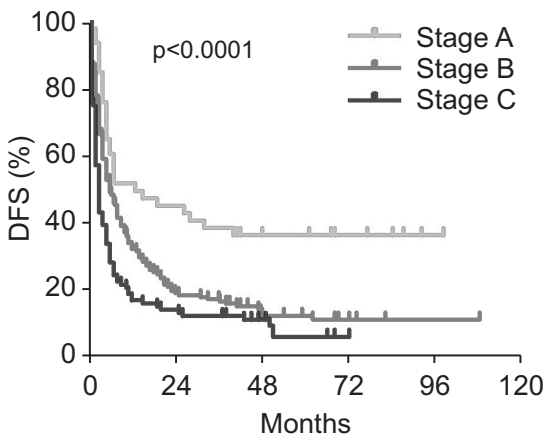

D JIS

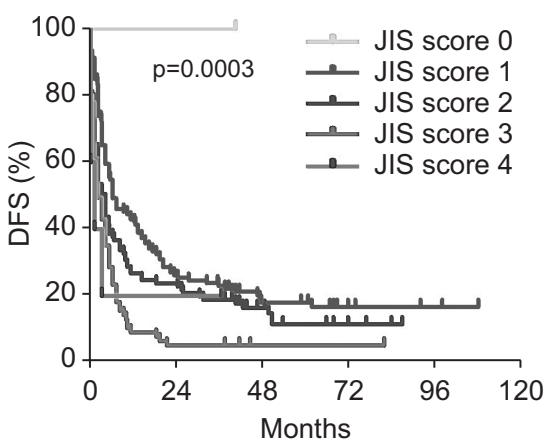

G BCLC

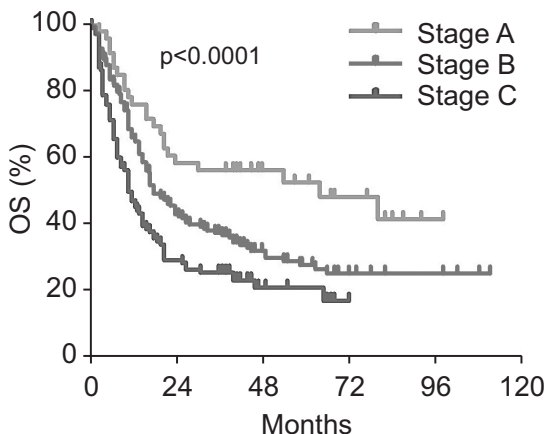

J JIS

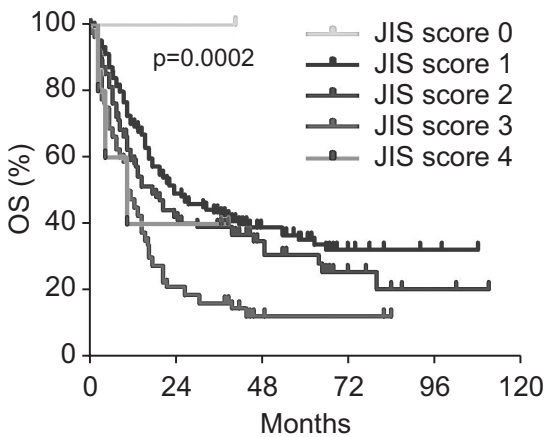

B AJCC

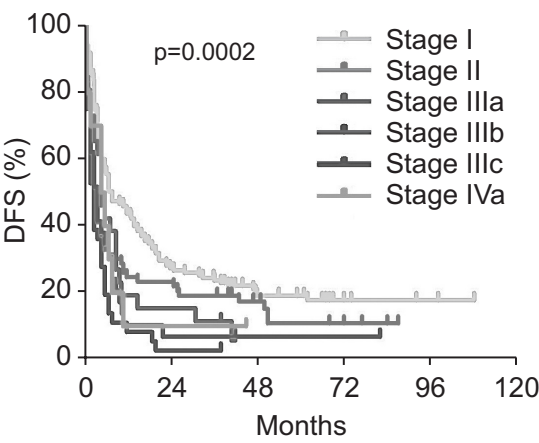

E okuda

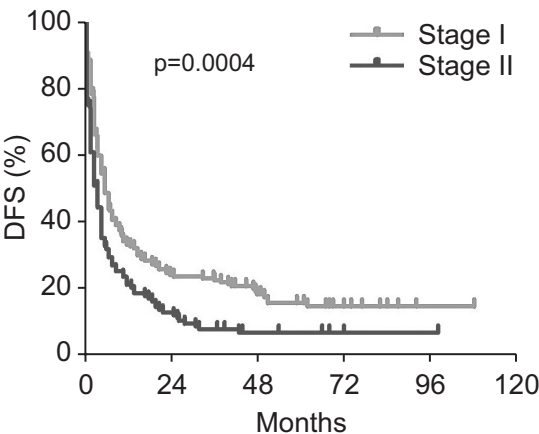

$\mathrm{H}_{\text {AJCC }}$

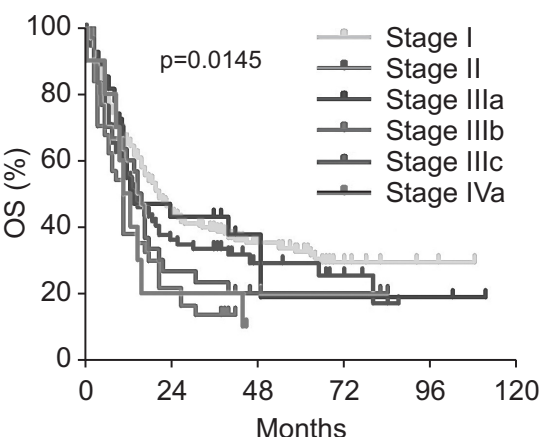

K Okuda

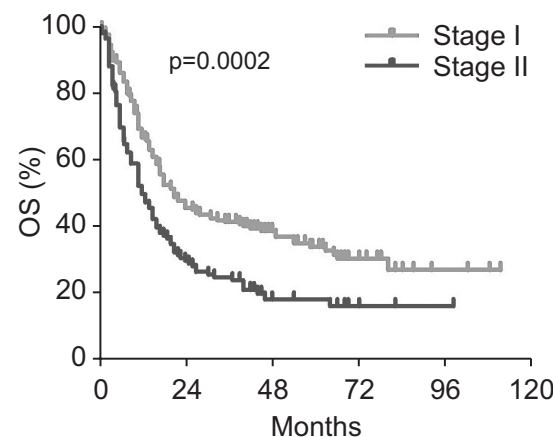

C CLIP

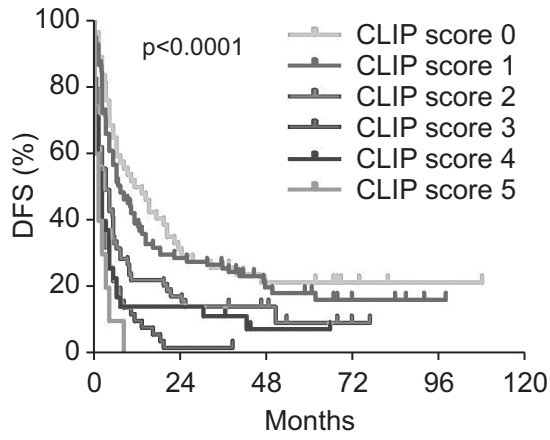

$\boldsymbol{F}_{\text {HKLC }}$

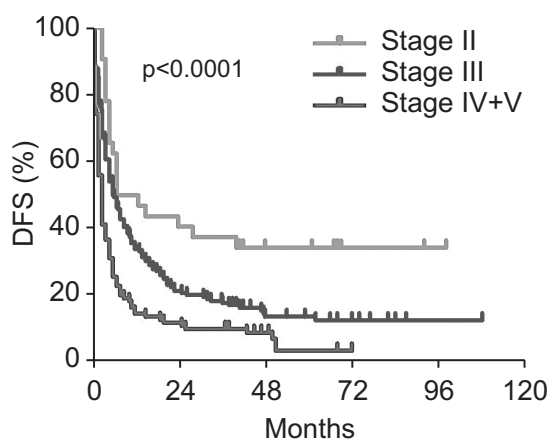

I CLIP

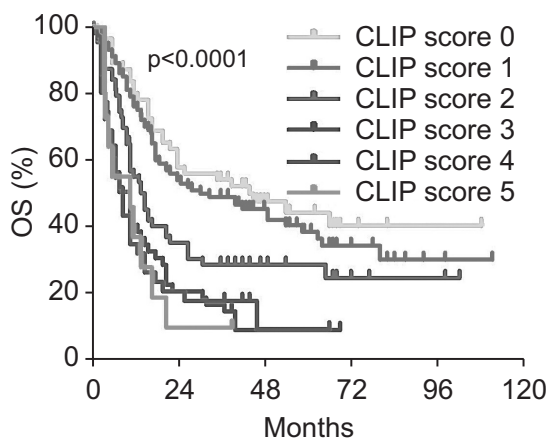

L HKLC

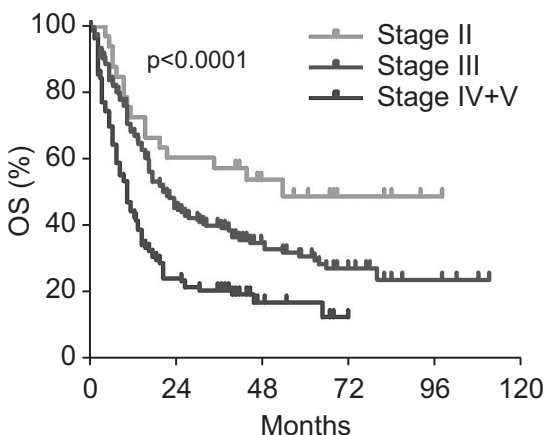

Fig. 5. Kaplan-Meier estimates of the prognosis of all included patients using conventional hepatocellular carcinoma staging systems. Disease-free survival (DFS) estimated by the Barcelona Clinic Liver Cancer (BCLC) staging system (A), the American Joint Committee on Cancer (AJCC) 2010 edition (B), the Cancer of the Liver Italian Program (CLIP) staging system (C), the Japan Integrated Staging Score (JIS) (D), the Okuda staging system (E), and the Hong Kong Liver Cancer (HKLC) prognostic classification scheme (F). Overall survival (OS) estimated by the BCLC staging system (G), the AJCC 2010 edition (H), the CLIP staging system (I), the JIS (J), the Okuda staging system (K), and the HKLC prognostic classification scheme (L). 


\section{ACKNOWLEDGEMENTS}

This study was supported by the grants of National Natural Science Foundation of China (number: 71673193), the Key Technology Research and Development Program of the Sichuan Province (numbers: 2015 SZ0131 and 2017FZ0082), and the Natural Science Foundation for Young Scientists and the Science \& Technology Planning Project of Gansu Province (number: 18JR3RA058).

\section{AUTHOR CONTRIBUTIONS}

Design this study: M.X., M.Z., L.Y. Data collection and analysis: L.X., L.L., P.W., Y.Z., X.H. Writing - original draft: L.X., T.W., B.L. Writing - review and editing: M.X. Approval of final manuscript: all authors.

\section{ORCID}

Liangliang $\mathrm{Xu}$

Lian Li

Peng Wang

Ming Zhang

Yanfang Zhang

Xiangyong Hao

Lvnan Yan

Bo Li

Tianfu Wen

Mingqing Xu https://orcid.org/0000-0002-3900-9972

https://orcid.org/0000-0002-8517-4661

https://orcid.org/0000-0001-5889-1115 https://orcid.org/0000-0002-8276-691X https://orcid.org/0000-0001-7614-0067 https://orcid.org/0000-0003-4419-7047 https://orcid.org/0000-0003-1371-8903 https://orcid.org/0000-0001-6572-5633 https://orcid.org/0000-0002-8260-7601 https://orcid.org/0000-0002-8556-0802

\section{REFERENCES}

1. Torre LA, Bray F, Siegel RL, Ferlay J, Lortet-Tieulent J, Jemal A. Global cancer statistics, 2012. CA Cancer J Clin 2015;65:87-108.

2. Befeler AS, Di Bisceglie AM. Hepatocellular carcinoma: diagnosis and treatment. Gastroenterology 2002;122:1609-1619.

3. Bismuth H, Majno PE, Adam R. Liver transplantation for hepatocellular carcinoma. Semin Liver Dis 1999;19:311-322.

4. Cha CH, Ruo L, Fong Y, et al. Resection of hepatocellular carcinoma in patients otherwise eligible for transplantation. Ann Surg 2003;238:315-321.

5. Kim H, Ahn SW, Hong SK, et al. Survival benefit of liver resection for Barcelona Clinic Liver Cancer stage B hepatocellular carcinoma. Br J Surg 2017;104:1045-1052.

6. Kokudo T, Hasegawa K, Matsuyama Y, et al. Survival benefit of liver resection for hepatocellular carcinoma associated with portal vein invasion. J Hepatol 2016;65:938-943.

7. Bruix J, Llovet JM. Prognostic prediction and treatment strategy in hepatocellular carcinoma. Hepatology 2002;35:519-524.

8. Grazi GL, Ercolani G, Pierangeli F, et al. Improved results of liver resection for hepatocellular carcinoma on cirrhosis give the procedure added value. Ann Surg 2001;234:71-78.
9. Rodríguez-Perálvarez M, Luong TV, Andreana L, Meyer T, Dhillon AP, Burroughs AK. A systematic review of microvascular invasion in hepatocellular carcinoma: diagnostic and prognostic variability. Ann Surg Oncol 2013;20:325-339.

10. Feng LH, Dong H, Lau WY, et al. Novel microvascular invasionbased prognostic nomograms to predict survival outcomes in patients after R0 resection for hepatocellular carcinoma. J Cancer Res Clin Oncol 2017;143:293-303.

11. Lei Z, Li J, Wu D, et al. Nomogram for preoperative estimation of microvascular invasion risk in hepatitis B virus-related hepatocellular carcinoma within the Milan criteria. JAMA Surg 2016;151:356-363.

12. Kaibori M, Ishizaki M, Matsui K, Kwon AH. Predictors of microvascular invasion before hepatectomy for hepatocellular carcinoma. J Surg Oncol 2010;102:462-468.

13. Wang CC, Iyer SG, Low JK, et al. Perioperative factors affecting longterm outcomes of 473 consecutive patients undergoing hepatectomy for hepatocellular carcinoma. Ann Surg Oncol 2009;16:1832-1842.

14. Fan ST, Poon RT, Yeung C, et al. Outcome after partial hepatectomy for hepatocellular cancer within the Milan criteria. Br J Surg 2011;98:1292-1300.

15. Roayaie S, Blume IN, Thung SN, et al. A system of classifying microvascular invasion to predict outcome after resection in patients with hepatocellular carcinoma. Gastroenterology 2009;137:850-855.

16. Shen J, Wen T, Chen W, Lu C, Yan L, Yang J. Model predicting the microvascular invasion and satellite lesions of hepatocellular carcinoma after hepatectomy. ANZ J Surg 2018;88:E761-E766.

17. Cho CS, Gonen M, Shia J, et al. A novel prognostic nomogram is more accurate than conventional staging systems for predicting survival after resection of hepatocellular carcinoma. J Am Coll Surg 2008;206:281-291.

18. Liang W, Zhang L, Jiang G, et al. Development and validation of a nomogram for predicting survival in patients with resected nonsmall-cell lung cancer. J Clin Oncol 2015;33:861-869.

19. Wang Y, Li J, Xia Y, et al. Prognostic nomogram for intrahepatic cholangiocarcinoma after partial hepatectomy. J Clin Oncol 2013;31:1188-1195.

20. Makuuchi M, Hasegawa H, Yamazaki S. Ultrasonically guided subsegmentectomy. Surg Gynecol Obstet 1985;161:346-350.

21. Makuuchi M, Torzilli G, Machi J. History of intraoperative ultrasound. Ultrasound Med Biol 1998;24:1229-1242.

22. Zhou L, Rui JA, Ye DX, Wang SB, Chen SG, Qu Q. EdmondsonSteiner grading increases the predictive efficiency of TNM staging for long-term survival of patients with hepatocellular carcinoma after curative resection. World J Surg 2008;32:1748-1756.

23. Evidence-based practice guidelines for standardized pathological diagnosis of primary liver cancer in China: 2015. Zhonghua Gan Zang Bing Za Zhi 2015;23:321-327.

24. Hou YF, Li B, Wei YG, et al. Second hepatectomy improves survival in patients with microvascular invasive hepatocellular carcinoma meeting the Milan criteria. Medicine (Baltimore) 2015;94:e2070 
25. Zhou J, Sun HC, Wang Z, et al. Guidelines for diagnosis and treatment of primary liver cancer in China (2017 Edition). Liver Cancer 2018;7:235-260.

26. Nathan H, Schulick RD, Choti MA, Pawlik TM. Predictors of survival after resection of early hepatocellular carcinoma. Ann Surg 2009;249:799-805.

27. Yeh CN, Chen MF, Lee WC, Jeng LB. Prognostic factors of hepatic resection for hepatocellular carcinoma with cirrhosis: univariate and multivariate analysis. J Surg Oncol 2002;81:195-202.

28. Izumi R, Shimizu K, Ii T, et al. Prognostic factors of hepatocellular carcinoma in patients undergoing hepatic resection. Gastroenterology 1994;106:720-727.

29. Belli G, Fantini C, Belli A, Limongelli P. Laparoscopic liver resection for hepatocellular carcinoma in cirrhosis: long-term outcomes. Dig Surg 2011;28:134-140.

30. Fu YP, Yi Y, Huang JL, et al. Prognostic nomograms stratify survival of patients with hepatocellular carcinoma without portal vein tumor thrombosis after curative resection. Oncologist 2017;22:561-569.

31. Nagao T, Inoue S, Yoshimi F, et al. Postoperative recurrence of hepatocellular carcinoma. Ann Surg 1990;211:28-33.

32. Chiappa A, Zbar AP, Audisio RA, Leone BE, Biella F, Staudacher C. Factors affecting survival and long-term outcome in the cirrhotic patient undergoing hepatic resection for hepatocellular carcinoma. Eur J Surg Oncol 2000;26:387-392.

33. Zeng QA, Qiu J, Hong J, et al. Hepatectomy for hepatocellular carcinoma patients with macronodular cirrhosis. Eur J Gastroenterol Hepatol 2012;24:575-582.

34. Faber W, Stockmann M, Schirmer C, et al. Significant impact of patient age on outcome after liver resection for HCC in cirrhosis. Eur J Surg Oncol 2014;40:208-213.

35. Huang J, Li BK, Chen GH, et al. Long-term outcomes and prognostic factors of elderly patients with hepatocellular carcinoma undergoing hepatectomy. J Gastrointest Surg 2009;13:1627-1635.

36. Motoyama H, Kobayashi A, Yokoyama T, et al. Impact of advanced age on the short- and long-term outcomes in patients undergoing hepatectomy for hepatocellular carcinoma: a single-center analysis over a 20-year period. Am J Surg 2015;209:733-741.

37. Nishikawa H, Arimoto A, Wakasa T, Kita R, Kimura T, Osaki Y. Surgical resection for hepatocellular carcinoma: clinical outcomes and safety in elderly patients. Eur J Gastroenterol Hepatol 2013;25:912-919.

38. Du Y, Cao GW. Challenges of incorporating gene expression data to predict HCC prognosis in the age of systems biology. World J Gastroenterol 2012;18:3941-3944.

39. Yan H, Yang Y, Zhang L, et al. Characterization of the genotype and integration patterns of hepatitis B virus in early- and lateonset hepatocellular carcinoma. Hepatology 2015;61:1821-1831.

40. Guan Y, Kuo WL, Stilwell JL, et al. Amplification of PVT1 contributes to the pathophysiology of ovarian and breast cancer. Clin Cancer Res 2007;13:5745-5755.

41. Shtivelman E, Bishop JM. The PVT gene frequently amplifies with
MYC in tumor cells. Mol Cell Biol 1989;9:1148-1154.

42. Carramusa L, Contino F, Ferro A, et al. The PVT-1 oncogene is a Myc protein target that is overexpressed in transformed cells. $\mathrm{J}$ Cell Physiol 2007;213:511-518.

43. Xu S, Kang CH, Gou X, et al. Quantification of liver fibrosis via second harmonic imaging of the Glisson's capsule from liver surface. J Biophotonics 2016;9:351-363.

44. Roan E. The effect of Glisson's capsule on the superficial elasticity measurements of the liver. J Biomech Eng 2010;132:104504.

45. Masaoka A, Monden Y, Nakahara K, Tanioka T. Follow-up study of thymomas with special reference to their clinical stages. Cancer 1981;48:2485-2492.

46. Nomori $\mathrm{H}$, Watanabe $\mathrm{K}$, Ohtsuka T, et al. Pulmonary metastasis 12 years after resection of thymoma with microscopic capsule invasion. Jpn J Clin Oncol 2004;34:630-633.

47. Rivera M, Ricarte-Filho J, Tuttle RM, et al. Molecular, morphologic, and outcome analysis of thyroid carcinomas according to degree of extrathyroid extension. Thyroid 2010;20:1085-1093.

48. Rosai J, Kuhn E, Carcangiu ML. Pitfalls in thyroid tumour pathology. Histopathology 2006;49:107-120.

49. Furlan JC, Bedard YC, Rosen IB. Significance of tumor capsular invasion in well-differentiated thyroid carcinomas. Am Surg 2007;73:484-491.

50. Hay ID, Bergstralh EJ, Goellner JR, Ebersold JR, Grant CS. Predicting outcome in papillary thyroid carcinoma: development of a reliable prognostic scoring system in a cohort of 1779 patients surgically treated at one institution during 1940 through 1989. Surgery 1993;114:1050-1057

51. Mazzaferri EL. Thyroid remnant 131I ablation for papillary and follicular thyroid carcinoma. Thyroid 1997;7:265-271.

52. Yoshida J, Nagai K, Asamura H, et al. Visceral pleura invasion impact on non-small cell lung cancer patient survival: its implications for the forthcoming TNM staging based on a large-scale nation-wide database. J Thorac Oncol 2009;4:959-963.

53. Manac'h D, Riquet M, Medioni J, Le Pimpec-Barthes F, Dujon A, Danel C. Visceral pleura invasion by non-small cell lung cancer: an underrated bad prognostic factor. Ann Thorac Surg 2001;71:1088-1093.

54. Huang XZ, Chen WJ, Zhang X, et al. An elevated platelet-tolymphocyte ratio predicts poor prognosis and clinicopathological characteristics in patients with colorectal cancer: a meta-analysis. Dis Markers 2017;2017:1053125.

55. Koh CH, Bhoo-Pathy N, Ng KL, et al. Utility of pre-treatment neutrophil-lymphocyte ratio and platelet-lymphocyte ratio as prognostic factors in breast cancer. Br J Cancer 2015;113:150-158.

56. Lai Q, Castro Santa E, Rico Juri JM, Pinheiro RS, Lerut J. Neutrophil and platelet-to-lymphocyte ratio as new predictors of dropout and recurrence after liver transplantation for hepatocellular cancer. Transpl Int 2014;27:32-41.

57. He X, Li JP, Liu XH, et al. Prognostic value of C-reactive protein/ albumin ratio in predicting overall survival of Chinese cervical cancer patients overall survival: comparison among various in- 
flammation based factors. J Cancer 2018;9:1877-1884.

58. Bacha S, Sghaier A, Habibech S, et al. Combined C-reactive protein and Neutrophil to Lymphocyte ratio use predict survival innon-small-cell lung cancer. Tunis Med 2017;95:229-235.

59. Bambace NM, Holmes CE. The platelet contribution to cancer progression. J Thromb Haemost 2011;9:237-249.

60. Senzel L, Gnatenko DV, Bahou WF. The platelet proteome. Curr Opin Hematol 2009;16:329-333.
61. Sarrouilhe D, Clarhaut J, Defamie N, Mesnil M. Serotonin and cancer: what is the link? Curr Mol Med 2015;15:62-77.

62. Nieswandt B, Hafner M, Echtenacher B, Männel DN. Lysis of tumor cells by natural killer cells in mice is impeded by platelets. Cancer Res 1999;59:1295-1300.

63. Unitt E, Marshall A, Gelson W, et al. Tumour lymphocytic infiltrate and recurrence of hepatocellular carcinoma following liver transplantation. J Hepatol 2006;45:246-253. 